\title{
Top electroweak couplings study at the ILC
}

\author{
Yo Sato* \\ Tohoku University \\ E-mail: vosatodepx.phys.tohoku.ac.7p
}

The top-quark electroweak couplings are expected to be strongly coupled to the mechanism of electroweak symmetry breaking and thus to be good probes for physics beyond the Standard Model. Currently these couplings can be measured only at hadron colliders, while a previous study shows an $e^{+} e^{-}$collider with beam polarizations, namely ILC, can improve the measurement accuracy which allows to probe new physics models. In order to extend the previous study at the ILC, we develop a new method to measure $t \bar{t} Z / \gamma$ couplings using all kinematic information of the di-leptonic final state from the top-quark pair production. We assume the center-of-mass energy of $500 \mathrm{GeV}$, two different beam polarization setups, $\left(P_{e^{-}}, P_{e^{+}}\right)=(-0.8,+0.3),(+0.8,-0.3)$, and the integrated luminosity of $250 \mathrm{fb}^{-1}$ for each beam polarization. The conventional method, which is also used in the previous study, measures the total cross section and the forwardbackward asymmetry. On the other hand, the method developed in this study measures all reconstructed angular information of the final state particles. We found that this new method can improve the measurement accuracy of the couplings by order of $10 \%$, up to $40 \%$, compared to the previous study at the ILC.

XIV International Conference on Heavy Quarks and Leptons (HQL2018)

May 27- June 1, 2018

Yamagata Terrsa, Yamagata,Japan

${ }^{*}$ Speaker. 


\section{Introduction}

The top-quark is the heaviest particle in the Standard Model (SM). Its large mass implies that it is strongly coupled to the mechanism of the Electroweak Symmetry Breaking (EWSB). The top electroweak couplings are good probes to new physics behind the EWSB, such as composite models [山] and Randall-Sundrum models [[]].

The top-quark is so far produced only at hadron colliders, where the top-quark electroweak coupling information can be extracted by top pair production in association with an electroweak boson (see e.g. refs. [] [ , [, [1, 目]). The International Linear Collider (ILC), which is a proposed electron-positron collider, is expected to complement such measurements at hadron colliders. At the ILC, the $t \bar{t} Z / \gamma$ coupling can be measured by top pair production without concurrent QCD production. The ILC will provide polarized beams which can disentangle $t \bar{t} Z$ and $t \bar{t} \gamma$ couplings. A previous study that measures two observables, namely the total cross section of the $e^{+} e^{-} \rightarrow t \bar{t}$ process $\sigma_{t t}$ and the forward-backward asymmetry $A_{F B}$ in the two beam polarizations, shows that the precision expected at the ILC allows to determine the new physics models [ $[$ ].

Our study aims at extending the previous study so that we can use the full kinematics of the final state of $t \bar{t}$ which allows us to fit more than four parameters as opposed to up to four parameters simultaneously in the previous study. Although such a method using full kinematics should give intrinsically higher sensitivity than the one with just two observables, currently only the partonlevel analysis has been established [ए]]. We study the feasibility of the full kinematics method in a more realistic simulation.

In the following we assume an integrated luminosity of $250 \mathrm{fb}^{-1}$ for each beam polarization condition $\left(P_{e^{-}}, P_{e^{+}}\right)=(-0.8,+0.3),(+0.8,-0.3)$ collected at a center of mass energy of $500 \mathrm{GeV}$ with a detector concept, ILD, at the ILC. The signal events, the di-leptonic final state $e^{+} e^{-} \rightarrow t \bar{t} \rightarrow$ $b l^{+} v_{l} \bar{b} l^{-} \bar{v}_{l}$, and all SM background events are reconstructed using the ILD full detector simulation [圆, 团].

\section{Optimal variables for the form factor fitting}

The $t \bar{t} Z / \gamma$ vertex can be written as

$$
\Gamma_{\mu}^{t t Z / \gamma}\left(k^{2}, q, \bar{q}\right)=i e\left[\gamma_{\mu}\left(F_{1 V}^{Z / \gamma}\left(k^{2}\right)+\gamma_{5} F_{1 A}^{Z / \gamma}\left(k^{2}\right)\right)+\frac{\sigma_{\mu v}}{2 m_{t}}(q+\bar{q})^{v}\left(i F_{2 V}^{Z / \gamma}\left(k^{2}\right)+\gamma_{5} F_{2 A}^{Z / \gamma}\left(k^{2}\right)\right)\right](2.1)
$$

Note that these form factors in the SM have following values:

$$
F_{1 V}^{\gamma}=\frac{2}{3}, F_{1 A}^{\gamma}=0, F_{1 V}^{Z}=\frac{1}{4 s_{W} c_{W}}\left(1-\frac{8}{3} s_{W}^{2}\right), F_{1 A}^{Z}=-\frac{1}{4 s_{W} c_{W}},
$$

where $s_{W}=\sin \theta_{W}, c_{W}=\cos \theta_{W}$ with $\theta_{W}$ being the weak mixing angle, and the other form factors are zero at tree level. Hereafter we will discuss only about $F_{1 V}^{Z / \gamma}, F_{1 A}^{Z / \gamma}$ and $F_{2 V}^{Z / \gamma}$ to compare with the previous study.

The distribution of signal events can be described in the helicity formalism with the narrow width approximation for top-quark and $W$. We denote the helicity of particle $x$ by $\lambda_{x}$. The full matrix element of the signal process is composed of 3 types of matrix elements: 
- $M_{\lambda_{t} \lambda_{\bar{t}}}^{\lambda_{e^{-}} \lambda_{e^{+}}}\left(\cos \theta_{t}, \phi_{t}\right)$ : The matrix element of the $t \bar{t}$ production

- $M_{\lambda_{W^{+}} \lambda_{b}}^{\lambda_{t}}\left(\cos \theta_{W^{+}}, \phi_{W^{+}}\right)\left(M_{\lambda_{W^{-}} \lambda_{\bar{b}}}^{\lambda_{\bar{b}}}\left(\cos \theta_{W^{-}}, \phi_{W^{-}}\right)\right)$: The matrix element of the decay $t \rightarrow W^{+} b(\bar{t} \rightarrow$ $\left.W^{-} \bar{b}\right)$

- $M_{\lambda_{l^{+}} \lambda_{v}}^{\lambda_{W^{+}}}\left(\cos \theta_{l^{+}}, \phi_{l^{+}}\right)\left(M_{\lambda_{l^{-}} \lambda_{\bar{v}}}^{\lambda_{W^{-}}}\left(\cos \theta_{l^{-}}, \phi_{l^{-}}\right)\right)$: The matrix element of the decay $W^{+} \rightarrow l^{+} v\left(W^{-} \rightarrow\right.$ $\left.l^{-} \bar{v}\right)$

where the angles are defined in the rest frame of each parent particle. The full matrix element is then described as follows:

$$
\begin{aligned}
& \left|M^{\lambda_{e^{-}} \lambda_{e^{+}}\left(\cos \theta_{t}, \phi_{t}, \cos \theta_{W^{+}}, \phi_{W^{+}}, \cos \theta_{W^{-}}, \phi_{W^{-}}, \cos \theta_{l^{+}}, \phi_{l^{+}}, \cos \theta_{l^{-}}, \phi_{l^{-}}\right)}\right|^{2} \\
= & \sum_{\lambda_{b} \lambda_{\bar{b}} \lambda_{l^{+}} \lambda_{l^{-}} \lambda_{v} \lambda_{\bar{v}}}\left|\sum_{\lambda_{t} \lambda_{\bar{t}} \lambda_{W^{+}} \lambda_{W^{-}}} M_{\lambda_{t} \lambda_{\bar{t}}}^{\lambda_{e^{-}} \lambda_{e^{+}}} M_{\lambda_{W^{+}} \lambda_{b}}^{\lambda_{t}} M_{\lambda_{l^{+}} \lambda_{v}}^{\lambda_{W^{+}}} M_{\lambda_{W^{-}} \lambda_{\bar{b}}}^{\lambda_{\bar{b}}} M_{\lambda_{l^{-}} \lambda_{\bar{v}}}^{\lambda_{W^{-}}}\right|^{2} .
\end{aligned}
$$

Left-handed helicities are only relevant for massless $b, l$ and $v$, and thus the first sum can be removed. The data analysis aims to probe the couplings between top and $Z / \gamma$. In the following we assume that the couplings of $W$ bosons are consistent with the SM. We expect that they will have been strongly constrained by the LHC by the time the ILC is realized.

In the following, $|M|^{2}$ represents the full matrix element squared for a given beam polarization condition. The expected number of events can be computed as a product of the total luminosity $L$ and the integration of $|M|^{2}$.

$$
N(\boldsymbol{F})=L \int|M(\boldsymbol{\Phi} ; \boldsymbol{F})|^{2} d \Phi
$$

where

$$
\begin{aligned}
\boldsymbol{F} & =\left(F_{1}, F_{2}, F_{3}, F_{4}, F_{5}, F_{6}, F_{7}, F_{8}, F_{9}, F_{10}\right) \\
& :=\left(F_{1 V}^{\gamma}, F_{1 V}^{Z}, F_{1 A}^{\gamma}, F_{1 A}^{Z}, F_{2 V}^{\gamma}, F_{2 V}^{Z}, \operatorname{Re} F_{2 A}^{\gamma}, \operatorname{Re} F_{2 A}^{Z}, \operatorname{Im} F_{2 A}^{\gamma}, \operatorname{Im} F_{2 A}^{Z}\right), \\
\boldsymbol{\Phi} & :=\left(\cos \theta_{t}, \cos \theta_{W^{+}}, \phi_{W^{+}}, \cos \theta_{W^{-}}, \phi_{W^{-}}, \cos \theta_{l^{+}}, \phi_{l^{+}}, \cos \theta_{l^{-}}, \phi_{l^{-}}\right) .
\end{aligned}
$$

$F_{2 A}$, which corresponds to the electric dipole moment term, can be a complex number. Since the system is invariant under an overall rotation around the $z$ axis, $\phi_{t}$ can be set as 0 and does not appear in $d \Phi$.

We define $\delta F_{i}$ and $\delta \boldsymbol{F}$ as deviations of the form factors from the values of the SM:

$$
\delta F_{i}:=F_{i}-F_{i, \mathrm{SM}}, \delta \boldsymbol{F}:=\boldsymbol{F}-\boldsymbol{F}_{\mathrm{SM}} .
$$

Expanding the matrix element around $\delta \boldsymbol{F}=\mathbf{0}$ gives

$$
|M(\boldsymbol{\Phi} ; \boldsymbol{F})|^{2}=\left(1+\sum_{i} \omega_{i}(\boldsymbol{\Phi}) \delta F_{i}+\sum_{i j} \tilde{\omega}_{i j}(\boldsymbol{\Phi}) \delta F_{i} \delta F_{j}\right)\left|M\left(\boldsymbol{\Phi} ; \boldsymbol{F}_{\mathrm{SM}}\right)\right|^{2},
$$

where the coefficients $\omega_{i}(\boldsymbol{\Phi})$ can be written as

$$
\omega_{i}(\boldsymbol{\Phi}):=\left.\frac{1}{\left|M\left(\boldsymbol{\Phi} ; \boldsymbol{F}_{\mathrm{SM}}\right)\right|^{2}} \cdot \frac{\partial|M(\boldsymbol{\Phi} ; \boldsymbol{F})|^{2}}{\partial F_{i}}\right|_{\boldsymbol{F}=\boldsymbol{F}_{\mathrm{SM}}} .
$$


Note that this $\omega_{i}$ is always computable once $\boldsymbol{\Phi}$ is obtained by reconstructing events. Taking $|M|^{2}$ in Eq. (R.8) as a function of $\omega_{i}(i=1,2, \ldots, 10)$, it is clear that the $\omega_{i}$ is the optimal variable to measure each $F_{i}$ in the nine-dimension Lorentz invariant phase space. We measure $\omega_{i}(\boldsymbol{\Phi})$ for each event and make a histogram of $\omega_{i}(\boldsymbol{\Phi})$, which basically has the same information as $\left|M\left(\omega_{i}\right)\right|^{2}$ and thus allows us to measure $\delta F_{i}$ by fitting the histogram.

\section{Signal and Background}

The $t \bar{t}$ process can be classified into three modes depending on the decays of $W$ bosons. The di-leptonic final state of $t \bar{t}$ happens when both of $W^{+}$and $W^{-}$decay leptonically. The branching ratio of the di-leptonic final state is $10.3 \%$. The full kinematics of the final state including the neutrinos can be obtained from the momenta of the isolated charged leptons and the bottom-quark jets by using kinematic constraints. The constraints are composed of the initial state constraints and the mass constraints for the top-quarks and the $W$ bosons.

The most relevant background contributions are $Z Z$ and other final states of $t \bar{t}$. These events can have charged leptons and bottom-quarks. Single top production, such as $e^{+} e^{-} \rightarrow W^{+*} W^{-} \rightarrow$ $t \bar{b} W^{-}$, and $Z W^{+} W^{-}$have the same final state as the signal events. The interference between these processes and signal process is considered. The process of $\gamma \gamma \rightarrow$ hadrons is the main source of the overlay background.

We first find two leptons that are isolated from the other particles that would be identified as bottom-quark jets. Then the remaining particles are clustered into two jets corresponding to the bottom-quarks. B-tagging is performed after the jet clustering process with the LCFIPlus framework [U] which makes use of vertex information. Then we construct a likelihood function from the eight kinematic constraints to recover the six unknown parameters corresponding to the momentum of the neutrinos. The two remaining constraints are used to compute the energy of the missing-photon along the beam line and to correct the b-jet energy resolutions. The unknowns are obtained by maximizing the likelihood function and the maximum is used to select signal from background events. Figure $\square$ shows an example of the residual distribution of $\cos \theta_{t}$.

\section{Fit results and discussion}

We performed single parameter fits for each form factor by minimizing $\chi^{2} \mathrm{~s}$ defined as

$$
\chi^{2}\left(F_{i}\right)=\sum_{i b i n=1}^{n b i n}\left(\frac{n_{i b i n}^{\text {Test }}-n_{\text {ibin }}^{\text {sim }}\left(F_{i}\right)}{\sqrt{n_{i b i n}^{\text {Test }}}}\right)^{2},
$$

where $n_{i b i n}$ denotes entries at a bin of $i b i n$ in the histogram of $\omega_{i}$, and the superscripts, Test and sim, indicate measured data and simulated events, respectively. In this study, we used also simulated data for $n^{\text {Test }}$, which is independent of the sample for $n^{\text {sim }}$, as pseudo-measured data. Table $\square$ shows our result summary together with the results from the previous study.

Figure $\square$ shows an example of a $\omega$ distribution and Figure $B$ shows an example of a $\chi^{2}$ distribution with which we validated our fit. 


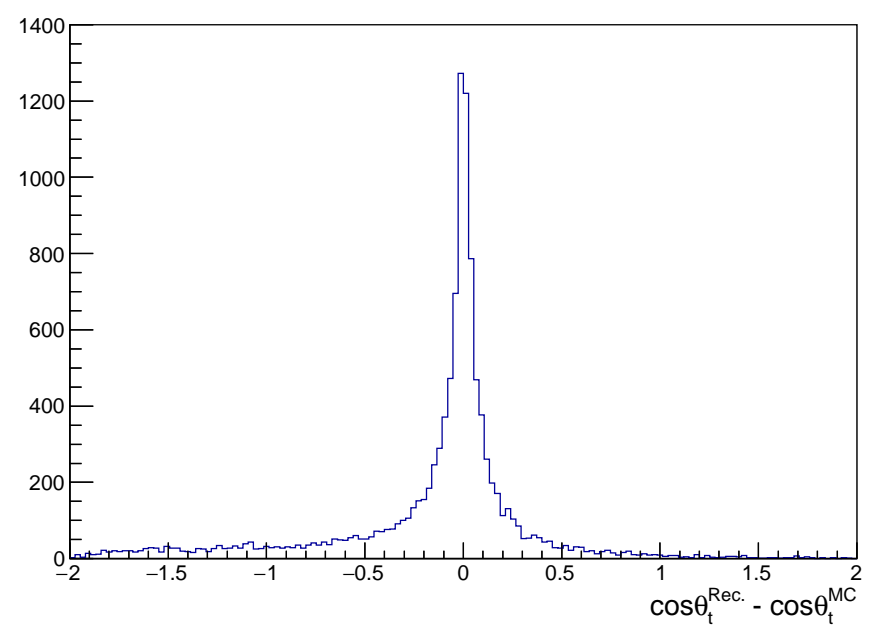

Figure 1: The distribution of difference between the reconstructed and true values of $\cos \theta_{t}$ in the $\left(P_{e^{-}}, P_{e^{+}}\right)=(-0.8,+0.3)$ case.

Table 1: Measurement accuracies of the form factors. The statistics of the previous study is approximately 6 times larger than that of this study. The third column is for comparison between this study and the previous study scaled with the statistics difference. In the previous study, $\left\{F_{1 V}^{\gamma}, F_{1 V}^{Z}, F_{1 A}^{Z}\right\}$ were fitted simultaneously assuming no variation of the other form factors. Similarly, $\left\{F_{2 V}^{\gamma}, F_{2 V}^{Z}\right\}$ were fitted simultaneously. $F_{1 A}^{\gamma}$ was fixed to be 0 in order to respect QED gauge invariance. We estimated the effects on the precision from the correlation between these parameters (see $[\square]$ ) to be at most $10 \%$.

\begin{tabular}{|c||c|c|c|}
\hline & this study (1) & previous study (2) & $(2) \times \sqrt{6}$ \\
\hline$F_{1 V}^{\gamma}$ & 0.0034 & 0.0016 & 0.0039 \\
\hline$F_{1 V}^{Z}$ & 0.0061 & 0.0028 & 0.0069 \\
\hline$F_{1 A}^{\gamma}$ & 0.0082 & - & - \\
\hline$F_{1 A}^{Z}$ & 0.013 & 0.0074 & 0.018 \\
\hline$F_{2 V}^{\gamma}$ & 0.0028 & 0.0013 & 0.0032 \\
\hline$F_{2 V}^{Z}$ & 0.0049 & 0.0022 & 0.0054 \\
\hline
\end{tabular}

This study and the previous one have common sources of systematic uncertainties, such as the integrated luminosity and the beam polarization. The previous study concluded these effects can be suppressed to be smaller than the statistical uncertainties. The experimental uncertainties in top-quark reconstruction and the beamstrahlung effects are also expected to cancel out in our analysis because our fitting is performed with templates that are produced with the full simulation including these effect. To make this assumption valid, however, a precise calibration between the simulation samples and the experimental data is necessary. This will be an additional component of the systematic uncertainties which should be estimated precisely. This issue is left for a future study. 


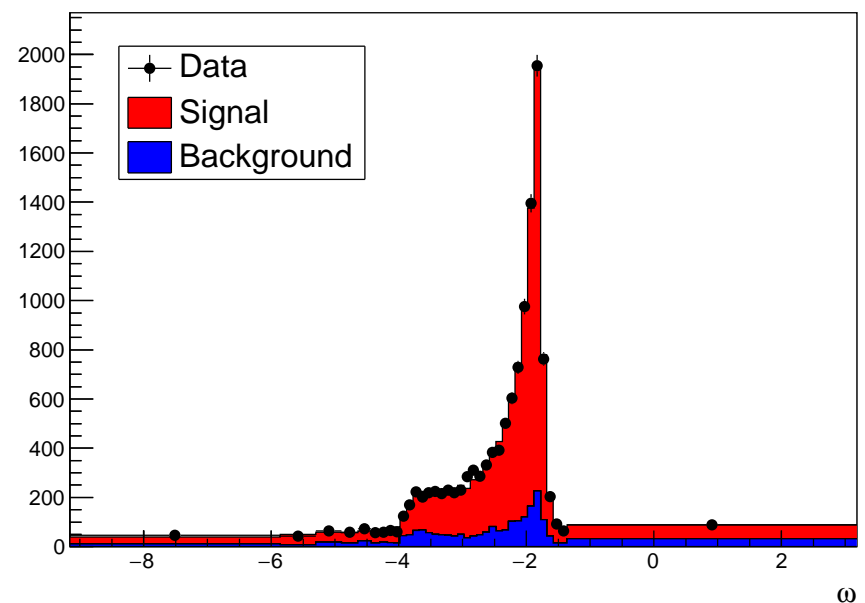

Figure 2: The distribution of $\omega_{1}$ which is the optimal variable of $F_{1}=F_{1 V}^{\gamma}$ in the $\left(P_{e^{-}}, P_{e^{+}}\right)=(-0.8,+0.3)$ case. The black dots show a pseudo-data (labeled as Data in the figure) and the filled histogram is the fitted distribution (red : signal events, blue : all SM background events).

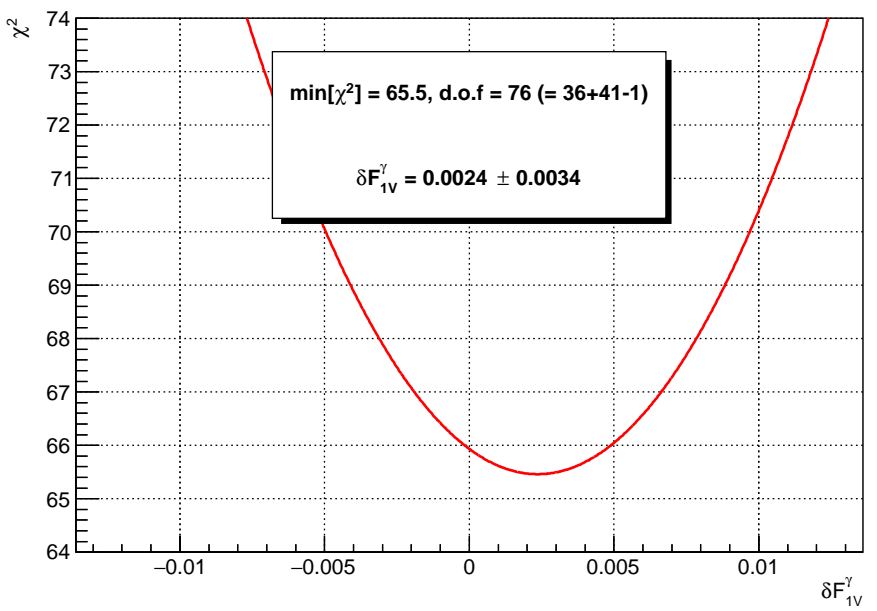

Figure 3: The plot of $\chi^{2}\left(\delta F_{1 V}^{\gamma}\right)$ function for the input of $\delta F_{1 V}^{\gamma}=0$. We validated our fit and obtained the statistical precision from this distribution.

\section{Conclusion and outlook}

The results are consistent with the previous study and show a possibility to improve the measurement accuracy compared to the method using the forward-backward asymmetry by order of $10 \%$, up to $40 \%$. The obtained results are based on single parameter fits. The multi-parameter fit, which is an advantage of this analysis, is addressed in ongoing studies.

The study presented in [12] shows precisions of the CP violating form factors $F_{2 A}^{Z / \gamma}$ with semileptonic final state. These parameters also can be measured in this analysis. A comparison between 
the two analyses is left for a future study.

\section{References}

[1] A. Pomarol and J. Serra, "Top Quark Compositeness: Feasibility and Implications”, Phys. Rev. D78 (2008) 074026, arXiv:0806.3247 [hep-ph].

[2] L. Randall and R. Sundrum, "A Large mass hierarchy from a small extra dimension", Phys. Rev. Lett. 83 (1999) 3370-3373, arXiv:hep-ph/9905221 [hep-ph].

[3] Aaboud, M. et al., " Measurement of the $t \bar{t} Z$ and $t \bar{t} W$ production cross sections in multilepton final states using $3.2 \mathrm{fb}^{-1}$ of $p p$ collisions at $\sqrt{s}=13 \mathrm{TeV}$ with the ATLAS detector", Eur. Phys. J. C 77 (2017) 40, https://doi.org/10.1140/epjc/s10052-016-4574-y

[4] Sirunyan, A.M. et al., "Measurement of the cross section for top quark pair production in association with a W or Z boson in proton-proton collisions at $\sqrt{s}=13$ TeV”, J. High Energ. Phys. 2017 (2017) 46, https://doi.org/10.1007/JHEP08(2017)046

[5] Sirunyan, A.M. et al., Measurement of the semileptonic $t \bar{t}+\gamma$ production cross section in pp collisions at $\sqrt{s}=8 \mathrm{TeV} J$. High Energ. Phys. 2017 (2017) 6. https://doi.org/10.1007/JHEP10(2017)006

[6] Aaboud, M. et al., J. High Energ. Phys. 2017 (2017) 86. https://doi.org/10.1007/JHEP11(2017)086

[7] M. S. Amjad et al., "A precise characterisation of the top quark electro-weak vertices at the ILC," Eur. Phys. J. C 75, no. 10, 512 (2015) doi:10.1140/epjc/s10052-015-3746-5, arXiv:1505.06020 [hep-ex].

[8] P. Mora de Freitas and H. Videau, "Detector simulation with MOKKA / GEANT4: Present and future", LC-TOOL-2003-010.

[9] F. Gaede, "Marlin and LCCD: Software tools for the ILC", Nucl. Instrum. Meth. A 559, 177 (2006). doi:10.1016/j.nima.2005.11.138.

[10] P. H. Khiem, E. Kou, Y. Kurihara and F. Le Diberder, "Probing New Physics using top quark polarization in the $e^{+} e^{-} \rightarrow t \bar{t}$ process at future Linear Colliders," arXiv:1503.04247 [hep-ph].

[11] T. Suehara and T. Tanabe, "LCFIPlus: A Framework for Jet Analysis in Linear Collider Studies", Nucl. Instrum. Meth. A 808, 109 (2016) doi:10.1016/j.nima.2015.11.054

[12] W. Bernreuther, L. Chen, I. García, M. Perelló, R. Poeschl, F. Richard, E. Ros and M. Vos, "CP-violating top quark couplings at future linear $e^{+} e^{-}$colliders," Eur. Phys. J. C 78, no. 2, 155 (2018) doi:10.1140/epjc/s10052-018-5625-3 [arXiv:1710.06737 [hep-ex]]. 